\title{
Bone marrow megakaryocytic activation predicts fibrotic evolution of Philadelphia-negative myeloproliferative neoplasms
}

\author{
Mattia Schino, ${ }^{1 *}$ Vincenzo Fiorentino,,$^{1 *}$ Elena Rossi, ${ }^{2,3}$ Silvia Betti, ${ }^{3}$ \\ Monica Di Cecca, ${ }^{2}$ Valentina Ranucci, ${ }^{1}$ Patrizia Chiusolo, ${ }^{2,3}$ \\ Maurizio Martini, ${ }^{1,3 \#}$ Valerio De Stefano ${ }^{2,3 \#}$ and Luigi Maria Larocca ${ }^{1,3 \#}$
}

Haematologica 2021

Volume 106(12):3162-3169

${ }^{1}$ Department of Life Sciences and Public Health, Università Cattolica del Sacro Cuore; ${ }^{2}$ Department of Radiological and Hematological Sciences, Università Cattolica del Sacro Cuore and ${ }^{3}$ Fondazione Policlinico Universitario A. Gemelli IRCCS, Rome, Italy

${ }^{*} M F$ and VF contributed equally as co-first authors.

\#VDS, MM and LML contributed equally as co-senior authors.

\section{ABSTRACT}

$\mathrm{P}$ hiladelphia-negative chronic myeloproliferative neoplasms (MPN) have been traditionally considered as indistinctly slowly progressing conditions; recent evidence proves that a subset of cases have a rapid evolution, so that MPN prognosis needs to be personalized. We identified a new morphological parameter, defined as megakaryocytic activation (M-ACT) based on the coexistence of megakaryocytic emperipolesis, megakaryocytes $(\mathrm{MK})$ cluster formation and evidence of arrangement of collagen fibers around the perimeter of $\mathrm{MK}$. We retrospectively analyzed the bone marrow biopsy of two MPN cohorts of patients with polycythemia (PV) $(n=64)$ and non-PV patients (including essential thrombocythemia, and early/prefibrotic primary myelofibrosis [PMF]) ( $\mathrm{n}=222)$. M-ACT showed a significant correlation with splenomegaly, white blood cell count, and lactate dehydrogenase serum levels in both groups, with JAK2 V617F allele burden in PV patients, and with CALR mutations, and platelet count in non-PV patients. Progression-free survival, defined as PV-to-secondary MF progression and non-PV-to-overt PMF, was worse in both PV and early/prefibrotic PMF patients with M-ACT in comparison to those without M-ACT $(P<0.0001)$. Interestingly, $\mathrm{M}$-ACT was not found in the subgroup of essential thrombocythemia patients. In conclusion, M-ACT can be helpful in the differential diagnosis of MPN and can represent a new morphologic parameter with a predictive value for progression of MPN.

Accepted: October 2, 2020.

Pre-published: November 19, 2020.

https://doi.org/10.3324/haematol.2020.264143

(C)2021 Ferrata Storti Foundation

Material published in Haematologica is covered by copyright. All rights are reserved to the Ferrata Storti Foundation. Use of published material is allowed under the following terms and conditions:

https://creativecommons.org/licenses/by-nc/4.0/legalcode. Copies of published material are allowed for personal or internal use. Sharing published material for non-commercial purposes is subject to the following conditions:

https://creativecommons.org/licenses/by-nc/4.0/legalcode, sect. 3. Reproducing and sharing published material for commercial purposes is not allowed without permission in writing from the publisher.

\section{Introduction}

Philadelphia-negative chronic myeloproliferative neoplasms (MPN) represent a group of hematological disorders that originates from the neoplastic transformation of a pluripotent stem cell and are characterized by clonal proliferation of one or more hematopoietic progenitors in the bone marrow (BM) and in extramedullary sites.

According to the World Health Organization (WHO) 2017 classification, MPN can be divided into three main sets: polycythemia vera (PV), essential phrombocythemia (ET) and primary myelofibrosis (PMF), whose early stages' differential diagnosis is often challenging. ${ }^{1}$

While MPN have been traditionally considered as indistinct slow progressing conditions, ${ }^{2,3}$ recent evidence, on the contrary, demonstrated that a subset of cases had a rapid evolution, leading different groups to develop several prognostic scores, mainly based on clinical and laboratory parameters with less emphasis on morphological, immunophenotypic and molecular data. ${ }^{4}$ The first prognostic score was the International Prognostic Scoring System (IPSS), edited in 2009 by an international 
study group, which enabled survival estimation at the time of diagnosis primarily employing five clinical and hematologic parameters; ${ }^{5}$ this model was further revised as the Dynamic International Prognostic Scoring System (DIPSS) and then as DIPSS-plus score. ${ }^{6,7}$ The above, nonetheless, applied to already-established myelofibrosis (both PMF and post-PV/ET MF) only, determining survival from the time of disease progression/transformation to death without considering the heterogeneous disease history before the appearance of BM changes. ${ }^{8}$

On the basis of advances in MPN molecular profiling, in order to improve the prognostic prediction in PMF patients, novel models included JAK2,CALR, and MPL mutation status in addition to the IPSS parameters. ${ }^{8}$ Moreover, novel insights were provided by in-depth analysis of genomic subsets with different clinical outcomes. ${ }^{9}$ Recent publications have introduced new risk models for PMF, namely MIPSS70 (mutation-enhanced international prognostic scoring system for transplant-age patients), ${ }^{10}$ MIPSS70+ version 2.0 (karyotype-enhanced MIPSS70) and GIPSS (genetically-inspired prognostic scoring system). ${ }^{11,12}$ Similar risk models have been recently introduced for both ET and PV under the name of MIPSS-ET and MIPSS-PV, highlighting the prognostic contribution of spliceosome gene mutations. ${ }^{13}$ However, all these predictive models do not consider morphological and phenotypical features, except BM fibrosis grade in the MIPPS70 model.

In this study we evaluated a new morphological parameter, defined by the coexistence of emperipolesis of megakaryocytes (MK) (i.e., the presence of an intact cell within the cytoplasm of another cell), MK clustering and peri-MK fibrosis in BM biopsy, which was named megakaryocytic activation (M-ACT). Larocca et al. in 2015 demonstrated that extensive BM emperipolesis associated to BM fibrosis was present in patients affected by gray platelet syndrome, with up to $65 \%$ MK containing two of four leukocytes engulfed within the cytoplasm; ${ }^{14}$ a similar phenomenon has been described either in BM patients with $\mathrm{PMF}^{15}$ and in the BM of animal models of myelofibrosis. $^{16,17}$

We demonstrated that M-ACT is a useful morphological parameter in forecasting both $\mathrm{PV}$ and early/prefibrotic PMF to myelofibrosis progression and could also help in the differential diagnosis between ET and early/prefibrotic PMF. ${ }^{18}$

\section{Methods}

\section{Patients' features}

Formalin-fixed, paraffin-embedded BM biopsy specimens, obtained from the posterior superior iliac spine, ${ }^{19}$ were available in our Institute of Pathology for 460 patients clinically diagnosed with a MPN and followed at our Institute of Hematology (Fondazione Policlinico Universitario "A. Gemelli", IRCCS) from January 2005 to October 2019. The study was carried out in accordance with the Declaration of Helsinki and the consent for retrospective analysis of all clinical data, according to the Ethical Committee of the Università Cattolica del Sacro Cuore School of Medicine, and obtained by all the patients at the hospital admission. Patients were clinically followed-up over the observation time by one single team physician (VDS and ER as senior members).

All 286 cases were sorted until October 2019, according to three inclusion criteria: clinical diagnosis of either PV or non-PV MPN, first BM biopsy at diagnosis for non-PV cohort and within 0-24 months from the clinical diagnosis for PV cohort and no grade 2-3 $\mathrm{BM}$ fibrosis. Accordingly, patients with diagnosis of overt PMF or secondary myelofibrosis were excluded. Furthermore, BM biopsies were revised by two skilled pathologists (LMR and MM) and categorized according to the WHO 2017 criteria (PV, ET, early/prefibrotic PMF).

Clinical and hematological data (according to WHO 2017 criteria) were collected in order to trace lactose dehydrogenase ( $\mathrm{LDH}$ ) increase (i.e., $\mathrm{LDH}$ serum levels $\geq 250 \mathrm{UI} / \mathrm{L}$ ), palpable splenomegaly, leukocytosis (i.e., white blood cell [WBC] count $\geq$ $11 \times 10^{9} / \mathrm{L}$ ), high hemoglobin $(\mathrm{Hg})$ level (i.e., $\mathrm{Hgb}>16 \mathrm{~g} / \mathrm{dL}$ for women and $\mathrm{Hgb}>16.5 \mathrm{~g} / \mathrm{dL}$ for men) and thrombocytosis (i.e., PLT $\geq 450 \times 10^{9} / \mathrm{L}$ ) for each patient at diagnosis. We also verified the occurrence of arterial/venous (A/V) thrombotic events and/or major bleeding events during the clinical course (until October 2019) for each case. Thrombotic and bleeding events were defined as previously described. ${ }^{20} J A K 2$ V617F mutation and allele burden analysis, CALR exon 9 mutations and MPL exon 10 mutations were performed as previously described. ${ }^{20}$ Progression to secondary myelofibrosis was defined from the patient chart review and based on the International Working Group for Myelofibrosis Research and Treatment (IWG-MRT) consensus criteria. ${ }^{21}$

The main clinical, hematological and molecular characteristics of the 286 patients are shown in Table 1 for the PV cohort (64 patients), in Table 2 and the Online Supplementary Table S1 for nonPV cohort (including 199 early/prefibrotic PMF patients [Table 2] and 23 ET patients [Online Supplementary Table S1]).

\section{Bone marrow biopsy analysis and megakaryocytic activation histological parameters}

All biopsy specimens had a suitable length (at least $1.5-2 \mathrm{~cm}$ ) in order to obtain at least ten partially preserved intertrabecular areas, since subcortical medullary lacunae are less cellular than deep ones (especially in the elderly) and since focal pathologies can have a deep localization. ${ }^{22}$ After collection, each biopsy specimen was kept in a properly-labeled clean container filled with $10 \%$ natural buffered formalin at $\mathrm{pH} 7.6$ for 12 hours for fixation, was then decalcified with a Decalcifier II solution (Leica Biosystems, Milan, Italy) for 1 hour at room temperature, then fixed with $10 \%$ natural buffered formalin at $\mathrm{pH} 7.6$ for 2 hours and finally embedded in paraffin. Sections (3-5 $\mu \mathrm{m}$ thick) were cut from each block for staining with hematoxylin and eosin (H\&E) and Gordon\&Sweet's silver staining to evaluate morphological features and fibrosis. ${ }^{23,24}$ The specimens were concurrently examined and reviewed by two pathologists experienced in BM biopsy interpretation (LML and MM), who were blinded toward the patients' characteristics and survival. Cases with disagreement were discussed using a multiheaded microscope until agreement was achieved. The agreement indices (Cohen's K) between the two pathologists were very good: $\mathrm{k}=0.83$ and $\mathrm{k}=0.85$ for $\mathrm{PV}$ group and for non-PV group, respectively.

In the definition of M-ACT the following parameters were examined in detail (as shown in Figure 1): (i) MK emperipolesis, (ii) $\mathrm{MK}$ clustering and (iii) peri-MK fibrosis: i) MK emperipolesis was defined as the presence of one or more leukocyte or a precursor of hematopoiesis within the cytoplasm of at least $30 \% \mathrm{MK}$ in the specimen; ii) MK clustering was defined as an aggregation of three or more megakaryocytes in close contact with each other and at least $25 \%$ of MK distributed in clusters in the specimen; iii) periMK fibrosis was defined as the arrangement of collagen fibers around the perimeter of the vast majority of $\mathrm{MK}$, underlining their primary role in the genesis of fibrosis.

M-ACT positive patients showed the contemporary presence of all three parameters and M-ACT was evaluated only on the first $\mathrm{BM}$ biopsy at diagnosis and before any treatment. 
Table 1. Correlation between megakaryocytic activation and the main polycythemia vera patient's clinical and molecular features.

\begin{tabular}{lccccc} 
PV & Overall $(n=64)$ & M-ACT $-(n=26)$ & M-ACT + (n=38) & HR [CI 95\%] & $P$ \\
Age $^{1}$ & $59.1(40-74)$ & $59.3(42-74)$ & $58.8(40-73)$ & $1.00[0.821-1.036]$ & 1.00 \\
Male & $38(59.4 \%)$ & $15(23.4 \%)$ & $23(35.9 \%)$ & $1.01[0.791-1.059]$ & 1.00 \\
\hline Female & $26(40.6 \%)$ & $11(17.2 \%)$ & $15(23.4 \%)$ & & \\
JAK2-RT > 50\% & $43(67.2 \%)$ & $16(25.0 \%)$ & $27(42.2 \%)$ & $1.24[0.058-2.589]$ & 0.059 \\
\hline Secondary MF-progression & $41(64.1 \%)$ & $6(9.4 \%)$ & $32(50 \%)$ & $2.87[0.332-1.591]$ & 0.0001 \\
Time-progression $^{2}$ & $78.3(26-116)$ & $95.5(72-116)$ & $44.7(26-48)$ & $2.78[0.036-2.589]$ & 0.0001 \\
\hline Hgb $^{3}$ & $18.7(15.2-19.9)$ & $16.2(15.2-18.4)$ & $17.1(15.4-19.9)$ & $1.31[0.228-2.746]$ & 0.06 \\
LDH $^{4}$ & $351.7(210-522)$ & $241.3(210-380)$ & $410.5(238-522)$ & $1.54[0.025-3.532]$ & 0.002 \\
\hline Palpable splenomegaly $^{\text {WBC }}{ }^{5}$ & $36(56.2 \%)$ & $16(25.0 \%)$ & $20(31.3 \%)$ & $1.83[0.179-3.795]$ & 0.001 \\
\hline PLT $^{6}$ & $11.3(7.2-20.5)$ & $9.8(7.2-13.4)$ & $15.6(10.3-20.5)$ & $1.81[0.054-3.273]$ & 0.001 \\
AN thrombosis & $590.8(380-851)$ & $507.2(380-623)$ & $720.4(430-851)$ & $2.39[0.082-2.629]$ & 0.0001 \\
\hline Major bleeding & $24(37.5 \%)$ & $8(12.5 \%)$ & $16(25.0 \%)$ & $1.02[0.882-1.069]$ & 0.43 \\
\hline
\end{tabular}

JAK2-RT: JAK2 V617F allele burden; Hgb: hemoglobin, serum levels; LDH: lactate dehydrogenase, serum levels; WBC: white blood cell count; PLT: platelet count; A/V: arterial/venous; HR: hazard ratio; CI: Confidence Interval. In years; ${ }^{2} \mathrm{In}$ months; ${ }^{3}(\mathrm{~g} / \mathrm{dL}) ;{ }^{4}(\mathrm{UI} / \mathrm{L}) ;{ }^{5}\left(\mathrm{x} 100^{9} / \mathrm{L}\right) ;{ }^{6}\left(\mathrm{x} 10^{9} / \mathrm{L}\right)$.

Table 2. Correlation between megakaryocytic activation and the main early/prefibrotic primary myelofibrosis patient's clinical and molecular features.

\begin{tabular}{|c|c|c|c|c|c|}
\hline Early/prefilorotic PMF & Overall $(n=199)$ & M-ACT - $(n=109)$ & M-ACT + $(n=90)$ & HR [Cl 95\%] & $P$ \\
\hline Age $^{1}$ & $66.2(46-78)$ & $64.2(48-76)$ & $66.3(46-78)$ & $1.01[0.67078-1.036]$ & 1.00 \\
\hline Male & $91(45.7 \%)$ & $57(28.6 \%)$ & $34(17.1 \%)$ & 1.04 [0.872-1.055] & 0.05 \\
\hline Female & $108(54.3 \%)$ & $52(26.7 \%)$ & $56(28.1 \%)$ & & \\
\hline JAK2-RT > 50\% & $37(18.6 \%)$ & $26(13.1 \%)$ & $11(5.5 \%)$ & 1.27 [0.073-2.879] & 0.04 \\
\hline CALR mut. & $60(30.1 \%)$ & $19(9.5 \%)$ & $41(20.6 \%)$ & 2.14 [0.301-1.902] & 0.001 \\
\hline CALR type 1 & $46(23.1 \%)$ & $11(5.5 \%)$ & $35(17.6 \%)$ & 3.01 [1.527-3.812] & 0.0001 \\
\hline CALR type 2 & $14(7.0 \%)$ & $8(4.0 \%)$ & $6(3.0 \%)$ & $0.97[0.833-1.056]$ & 1.00 \\
\hline MPL mut. & $3(1.5 \%)$ & $2(1.0 \%)$ & $1(0.5 \%)$ & $1.00[0.663-1.044]$ & 1.00 \\
\hline Time-progression ${ }^{2}$ & $67.3(15-109)$ & $70.2(53-109)$ & $33.7(15-56)$ & 2.83 [0.328-1.913] & 0.0001 \\
\hline $\mathrm{Hgb}^{3}$ & $15.3(12.8-16.4)$ & $14.8(12.8-15.5)$ & $15.2(13.6-16.4)$ & 1.04 [0.923-1.088] & 0.43 \\
\hline LDH serum levels $^{4}$ & $402.7(205-612)$ & $230.3(205-390)$ & $407.9(288-612)$ & 1.54 [0.045-3.235] & 0.003 \\
\hline Palpable splenomegaly & $111(55.8 \%)$ & $49(24.6 \%)$ & $62(31.1 \%)$ & $1.82[0.1797-3.759]$ & 0.001 \\
\hline $\mathrm{WBC}^{5}$ & $11.8(7.5-18.7)$ & $10.3(7.5-14.8)$ & $14.4(9.9-18.7)$ & $1.80[0.045-3.733]$ & 0.002 \\
\hline $\mathrm{PLT}^{6}$ & $620.1(366-861)$ & $520.6(366-650)$ & $710.3(490-861)$ & $2.46[0.087-2.279]$ & 0.0001 \\
\hline AN thrombosis & $74(37.2 \%)$ & $30(15.1 \%)$ & $44(22.1 \%)$ & 1.03 [0.243-1.912] & 0.001 \\
\hline Major bleeding & $30(15.1 \%)$ & $14(7.0 \%)$ & $16(8.0 \%)$ & 1.00 [0.928-1.079] & 0.42 \\
\hline
\end{tabular}

JAK2-RT: JAK2 V617F allele burden; CALR mut: CALR exon 9 mutations (type $1+$ type 2); MPL mut: MPL exon 10 mutations; Hgb: hemoglobin: serum levels; LDH: lactate dehydrogenase, serum levels; WBC: white blood cell count; PLT: platelet count; A/V: arterial/venous; HR: hazard ratio; CI: Confidence Interval. ${ }^{1}$ In years; ${ }^{2} \mathrm{In}$ months; ${ }^{3}(\mathrm{~g} / \mathrm{dL}){ }^{4}(\mathrm{UI} / \mathrm{L})$; ${ }^{5}\left(\mathrm{x} 10^{9} / \mathrm{L}\right) ;{ }^{6}\left(\mathrm{x} 10^{9} / \mathrm{L}\right)$

\section{Statistical analysis}

Statistical analysis was performed using GraphPad-Prism 5 software (Graph Pad Software, San Diego, CA) and MedCalc version 10.2.0.0 (MedCalc Software, Mariakerke, Belgium). ${ }^{25}$ Statistical comparison of continuous variables was performed by the MannWhitney $U$ test (t-test), as appropriate. Comparison of categorical variables was performed by $\chi^{2}$ statistic, using the Fisher's exact test. In order to evaluate the agreement between the two pathologists about the presence or absence of M-ACT in BM biopsies, the interrater agreement (Kappa) using MedCalc software was calulated.

The endpoint was progression-free survival (PFS), defined as the time between the first diagnosis and PV-to-secondary MF progression and early/prefibrotic PMF-to-overt PMF progression, respectively.

We followed the WHO 2017 criteria to establish the progression for PV-to-secondary MF and for early/prefibrotic PMF to overt myelofibrosis progression. ${ }^{1}$
Kaplan-Meier survival curves were plotted and differences in survival between groups of patients were compared using the logrank test. Multivariate analysis was performed using the Cox proportional hazards regression analysis including only those clinical and biological variables with a $P$-value of 0.10 or lower at the univariate analysis. $P$-values less than 0.05 were considered as statistically significant.

\section{Results}

\section{Megakaryocytic activation in the polycythemia vera cohort}

Twenty-six of the 64 PV did not meet histological criteria for M-ACT (40\%), versus 38 who did (60\%). In the PV cohort, M-ACT showed a significant correlation with one clinical parameter, i.e., palpable splenomegaly $(P=0.001)$, 


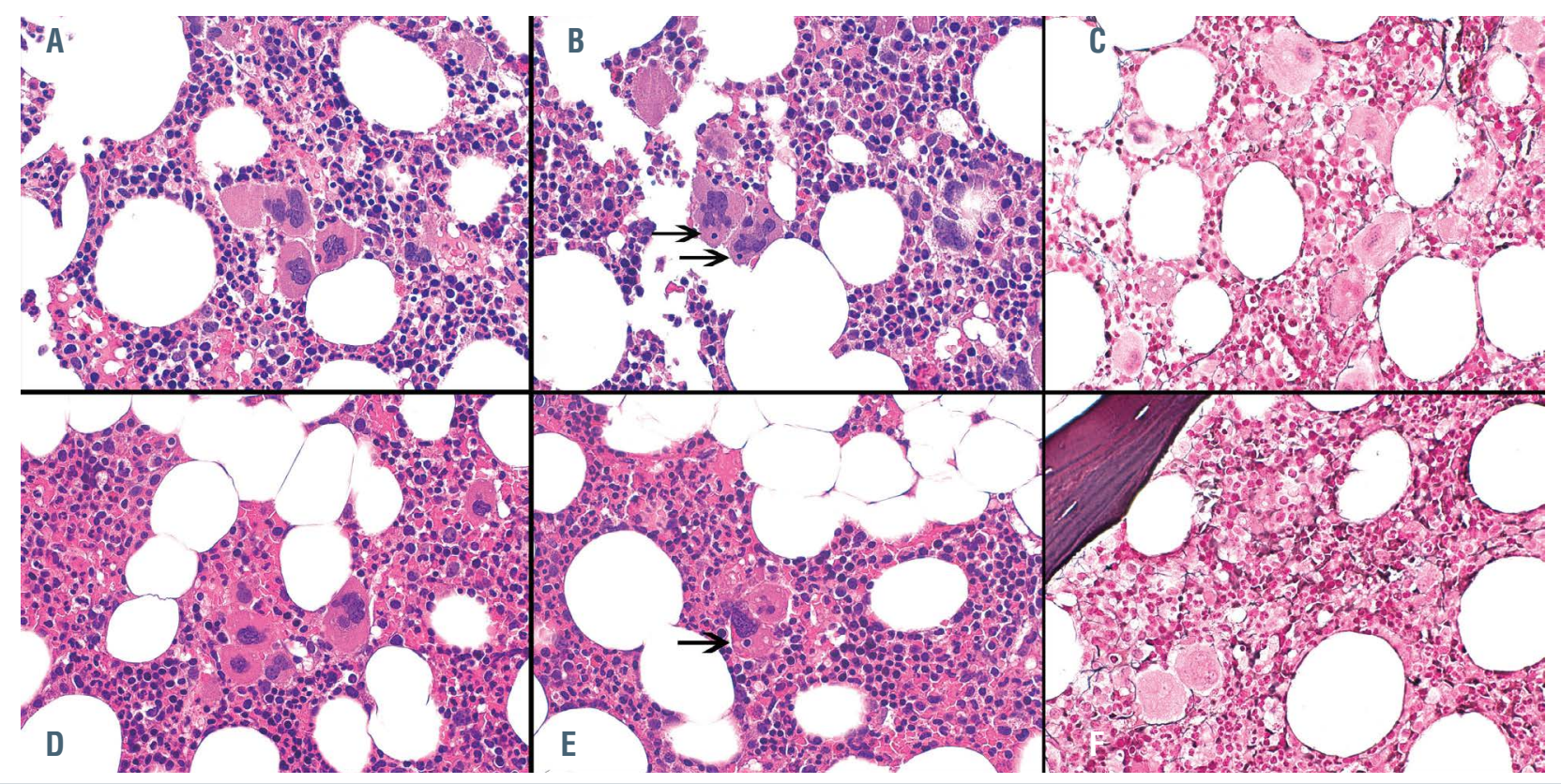

Figure 1. Representative images of megakaryocytic activation in polycythemia vera (A to C) and in early/prefibrotic primary myelofibrosis (D to F). (A and D) Emperipolesis, defined as the presence of at least one leukocyte (indicated by black arrows) or a precursor of hematopoiesis within the cytoplasm of a megakaryocyte (MK) (400X magnification); (B and E) MK clustering, defined as an aggregation of three or more MK in close contact with each other (200X and 400X magnification for panels $\mathrm{B}$ and $\mathrm{E}$, respectively); ( $\mathrm{C}$ and F) peri-MK fibrosis, defined as the arrangement of collagen fibers around the perimeter of activated MK (400X and 200X magnification for panel $C$ and $F$, respectively).

Table 3. Polycythemia vera cohort: progression-free survival multivariate analysis.

\begin{tabular}{lcccccc} 
Covariate & D & SE & Wald & $P$ & Exp $(\mathrm{b})$ & $95 \%$ Cl of Exp $(\mathrm{b})$ \\
Major bleeding & -0.1562 & 0.3890 & 0.1614 & 0.6879 & 0.8554 & 0.3991 to 1.8333 \\
JAK2 status & 1.0726 & 0.4191 & 6.5500 & 0.0105 & 2.9229 & 1.2855 to 6.6460 \\
\hline M-ACT & 2.3435 & 0.4761 & 24.2335 & $<0.0001$ & 10.4180 & 4.0978 to 26.4858 \\
WBC & -0.1520 & 0.3604 & 0.1780 & 0.6731 & 0.8590 & 0.4239 to 1.7407 \\
\hline
\end{tabular}

$b$ : coefficient estimates; $\mathrm{SE}$ : standard error for coefficient estimates b; Exp(b): hazard ratio value; $95 \% \mathrm{CI}$ of Exp(b)=95\% Confidence Interval of hazard ratio. Major bleeding $v$ s. no major bleeding; JAK2 burden $\geq 50 \%$ vs. JAK2 burden $<50 \%$ (JAK2 status); megakaryocyte activation vs. no megakaryocyte activation (M-ACT); WBC $\geq 11.0 \times 10^{9} / \mathrm{L}$ vs WBC $<11.0 \times 10^{9} / \mathrm{L} ;$ WBC: whitel blood cell count.

Table 4. Early/prefibrotic primary myelofibrosis subset: progression-free survival multivariate analysis.

\begin{tabular}{lcccccc} 
Covariate & $b$ & SE & Wald & $P$ & Exp(b) & $95 \%$ C of Exp(b) \\
WBC & 0.4334 & 0.1879 & 5.3207 & 0.0211 & 1.5425 & 1.0673 to 2.2294 \\
M-ACT & 0.7659 & 0.1640 & 21.8235 & $<0.0001$ & 2.1510 & 1.5598 to 2.9661 \\
\hline CALR status & 0.3690 & 0.1685 & 4.7956 & 0.0285 & 1.4463 & 1.0395 to 2.0124 \\
Sex & 0.4071 & 0.1520 & 7.1702 & 0.0074 & 1.5024 & 1.1153 to 2.0240 \\
LDH & 0.1831 & 0.1749 & 1.0958 & 0.2952 & 1.2009 & 0.8524 to 1.6919 \\
Palpable splenomegaly & 0.3084 & 0.1746 & 3.1217 & 0.0773 & 1.3613 & 0.9668 to 1.9167 \\
\hline PLT & 0.2415 & 0.1638 & 2.1729 & 0.1405 & 1.2731 & 0.9235 to 1.7551 \\
\hline
\end{tabular}

$b$ : coefficient estimates; SE: standard error for coefficient estimates b; $\operatorname{Exp}(\mathrm{b})$ : hazard ratio value; $95 \% \mathrm{CI}$ of Exp(b): $95 \%$ Confidence Interval of hazard ratio; WBC: white blood cell count; PLT: platelet count; $\mathrm{LDH}$ : lactose dehydrogenase; M-ACT: megakaryocyte activation. CALR mutations vs. no CARL wt (CARL); sex male vs. female (sex); megakaryocyte activation $v$ s. no megakaryocyte activation (M-ACT); WBC count $\geq 11.0 \times 10^{9} / \mathrm{L} v s$.: WBC $<11.0 \times 10^{9} / \mathrm{L} ; \mathrm{LDH} \geq 250 \mathrm{UI} / \mathrm{L} v \mathrm{~s}$. LDH<250 UI/L; palpable splenomegaly $v s$. no palpable splenomegaly; $\mathrm{PLT} \geq 450 \times 10^{9} / \mathrm{L}$ vs. PLT $<450 \times 10^{9} / \mathrm{L}$

and with hematologic parameters, like platelet count $(P=0.0001)$, LDH serum levels $(P=0.002)$ and WBC count $(P=0.001)$. On the other hand, no significant correlation was found between M-ACT and age $(P=1.00)$, sex $(P=1.00), \mathrm{A} / \mathrm{V}$ thrombosis $(P=0.43)$, while major bleeding $(P=0.06)$, Hgb level $(P=0.06)$ and $J A K 2$ V617F burden $>50 \%$ $(P=0.059)$ showed a certain associative trend (Table 1$)$.

We found that patients with M-ACT had a significant lower PFS than those without M-ACT (Table 1; Figure 2 panel A, for PFS: median PFS for M-ACT positive patients
58 months vs. median PFS for M-ACT negative patients 108 months, $P<0.0001$, hazard ratio [HR] 6.81, 95\% Confidence Interval [CI]: 3.48-13.32). Moreover, JAK2 V617F allele burden $\geq 50 \%$ and history of major bleeding had a significant correlation with a worse PFS $(P=0.0225$ and $P=0.0174$, respectively, Online Supplementary Figure S1), while WBC count $>11 \times 10^{9} / \mathrm{L}$ showed a certain trend toward significance $(P=0.0823$, Online Supplementary Figure S1). Conversely, age $(P=0.3718)$, sex $(P=0.3645)$, $\mathrm{LDH}$ serum level $(P=0.1305)$, PLT count $(P=0.5643)$, Hgb level 
A

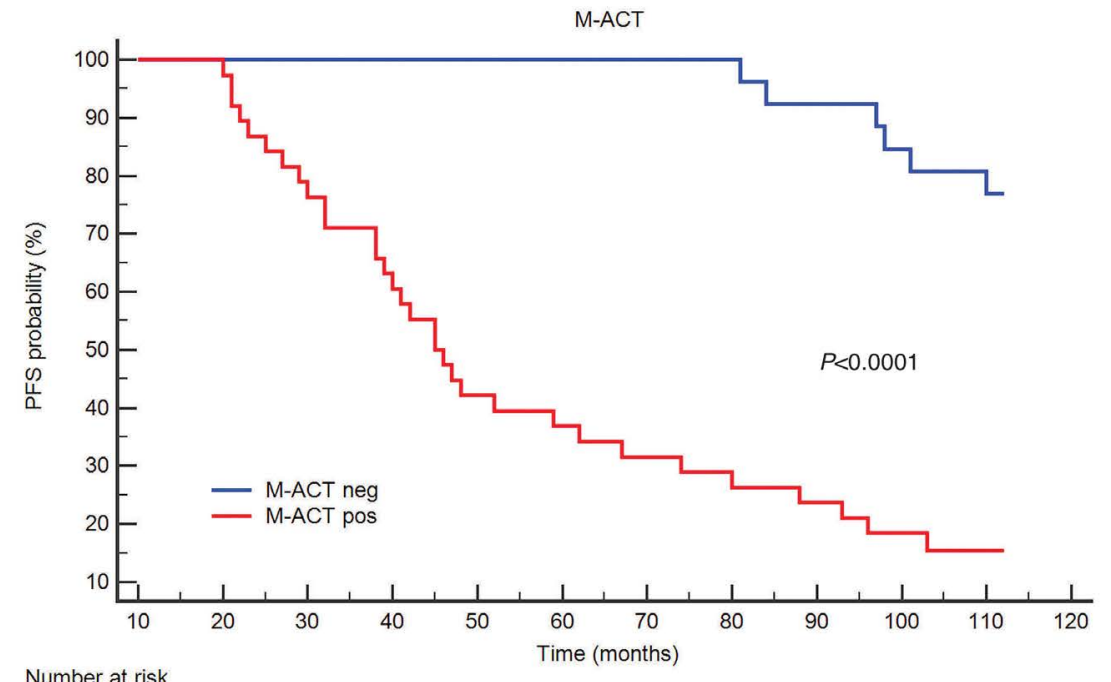

Number at risk

$\begin{array}{ccccccccccc}\text { Group: M-ACT neg } \\ \begin{array}{c}26 \\ 26\end{array} & 26 & 26 & 26 & 26 & 26 & 26 & 24 & 22 & 20 & 0 \\ \text { Group: M-ACT pos } \\ 38 \quad 37 & 29 & 23 & 16 & 14 & 12 & 10 & 9 & 6 & 3 & 0\end{array}$

B
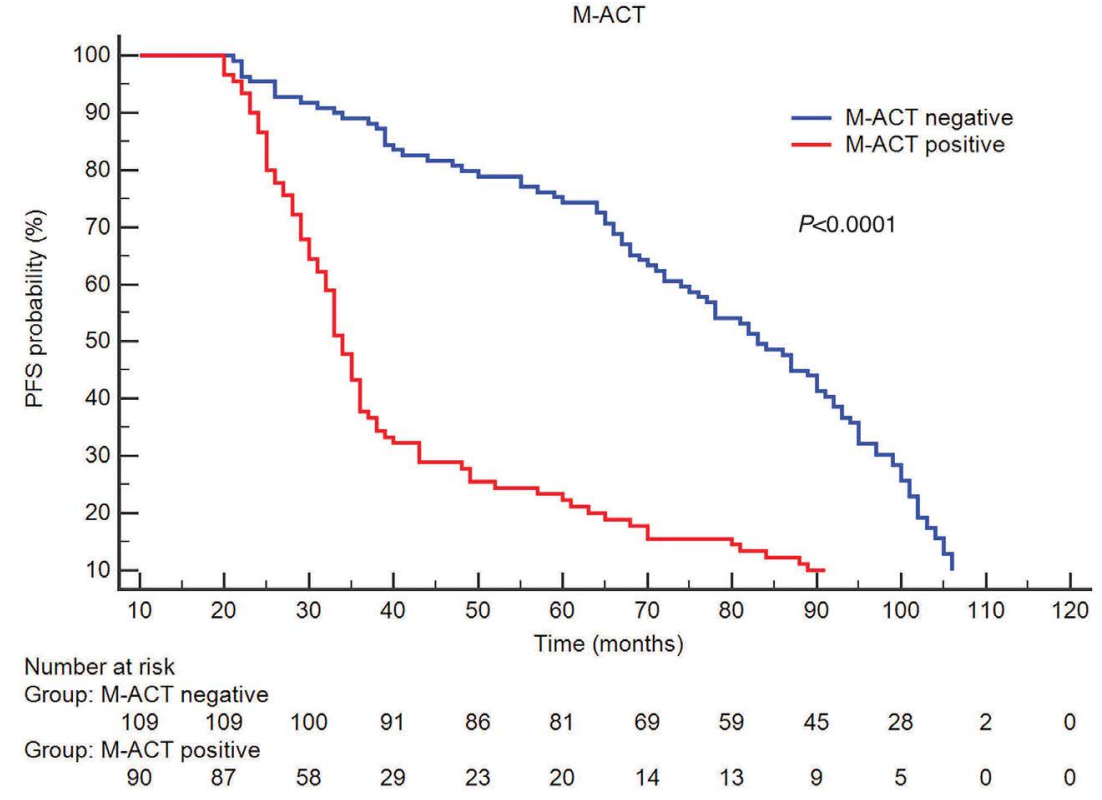

Figure 2. Kaplan-Meier curves for progression-free survival of polycythemia vera and of early/prefibrotic primary myelofibrosis patients. (A) Kaplan-Meier curve for progression-free survival (PFS) of polycythemia vera (PV) patients stratified by megakaryocytic activation (M-ACT); (B) Kaplan-Meier curve for PFS of early/prefibrotic primary myelofibrosis (PMF) patients by M-ACT. M-ACT positive patients (red-line) was significantly associated to a worse PFS $(P<0.0001)$ with respect to those without M-ACT (blue-line) in both groups.

$(P=0.1024)$ and $\mathrm{A} / \mathrm{V}$ thrombosis $(P=0.4216)$ did not show significant correlation with PFS.

Multivariate analysis of PFS, including M-ACT status, $J A K 2$ status, WBC count and history of major bleeding, showed that the presence of M-ACT and the JAK2 V617F allele burden were the only significant predictors (for M-ACT status, $P<0.0001$, HR 10.4180, 95\% CI: 4.097826.4858; for JAK2 V617F allele burden $\geq 50 \%, P=0.0105$, HR 0.0105, 95\% CI: 1.2855-6.6460; Table 3).

\section{Megakaryocytic activation in non-polycythemia vera myeloproliferative neoplasm cohort}

One hundred and nine of 199 early/prefibrotic PMF patients did not meet histological criteria for M-ACT
(55\%) versus 90 who did (45\%). In this cohort, M-ACT showed a strong correlation with clinical parameters, such as palpable splenomegaly $(P=0.001)$ and history of major bleeding $(P=0.001)$, and with hematologic parameters, like platelet count $(P=0.0001)$, LDH serum levels $(P=0.003)$, WBC count $(P=0.002)$, presence of CALR mutations $(P=0.001$; Table 2). Notably, we found a significant association between M-ACT and CALR type 1 mutation $(P=0.0001)$ while we did not find a significant correlation between M-ACT and CALR type 2 mutation $(P=1.0)$. We found a significant yet milder correlation with sex, with M-ACT being more prevalent in females $(P=0.05)$, and with JAK2 V617F allele burden $\geq 50 \% \quad(P=0.04)$. On the contrary, no significant correlation was found between 
M-ACT and age $(P=1.00)$, Hgb level $(P=0.43), M P L$ mutations $(P=1.00)$ and $\mathrm{A} / \mathrm{V}$ thrombosis $(P=0.42$; Table 1$)$.

Similarly to what happened in the PV cohort, when we correlated M-ACT status with PFS, we found that patients with early/prefibrotic PMF and with M-ACT had a significant lower PFS than those without M-ACT (Table 1; Figure 2 panel B, for PFS: median PFS for M-ACT positive patients 44 months $v s$. median PFS for M-ACT negative patients 77 months, $P<0.0001$, HR 3.17, 95\% CI: 2.27 4.44). Moreover, male sex, CALR type 1 mutations, WBC count $>11 \times 10^{9} / \mathrm{L}$, presence of palpable splenomegaly, $\mathrm{PLT} \geq 600 \times 10^{9} / \mathrm{L}$ and $\mathrm{LDH} \geq 250 \mathrm{U} / \mathrm{L}$ had a significant correlation with a worse PFS $(P=0.0187, P<0.0001, P<0.0001$, $P<0.0001, P<0.0001$ and $P=0.0025$, respectively, Online Supplementary Figure 2S). Conversely, age $(P=0.8831)$, major bleeding $(P=0.7244)$, JAK2 V617F allele burden $\geq$ $50 \%(P=0.3459)$, Hgb level $(P=0.5234), M P L$ mutations $(P=0.2268)$ and $\mathrm{A} / \mathrm{V}$ thrombosis $(P=0.2003)$ did not show significant correlation with PFS.

Multivariate analysis of PFS, including M-ACT status, CALR status, WBC count, sex, LDH serous level, splenomegaly, and platelet count, showed that the presence of M-ACT and CALR type 1 mutation, WBC count $>11 \times 10^{9} / \mathrm{L}$ and male sex were the significant predictors (for M-ACT status, $P<0.0001$, HR 2.1510, 95\% CI: $1.5598-$ 2.9661; for CALR status, $\mathrm{P}=0.0285$, HR 1.446 , $95 \% \mathrm{CI}$ : 1.0395-2.0124; for WBC count, $P=0.0211$, HR 1.5425, 95\% CI: 1.0673-2.2294; for sex, $P=0.0074$, HR 1.5024, 95\% CI: 1.1153-2.0240; Table 4).

In the non-PV MPN cohort, we also analyzed a small subgroup of 23 ET patients. We did not find M-ACT in any of the ET BM biopsies performed at the time of the diagnosis. The ET patients had a better PFS in comparison to patients with early/prefibrotic PMF either with M-ACT (Online Supplementary Figure S3; $P<0.0001$ ) and without MACT (Online Supplementary Figure S3; $P<0.0001$ ).

Interestingly, we found that the incidence of M-ACT among triple-negative patients in the non-PV cohort was significantly lower in respect to patients with a driver gene mutation (21 of $85[24.7 \%$ ] triple negative patients $v s .90$ of $222[40.5 \%] ; P=0.0115)$, and that triple-negative patients with M-ACT also had a significant lower PFS than those without M-ACT (median PFS for M-ACT positive triple-negative patients 56 months vs. median PFS for M-ACT negative triple-negative patients 79 months, $P<0.0023$, HR 2.76, 95\% CI: 1.44-5.32; data not shown).

\section{Discussion}

For about two decades, one of the most important problems in the treatment of patients with MPN has been the identification of biological and non-biological factors that could represent a determinant key to the prediction of prognosis. Accordingly, several prognostic scores have succeeded over time, mainly based on clinical, hematological and molecular parameters, in identifying the fraction of MPN patients that could have a high risk of developing a leukemic transformation or a bone marrow fibrotic failure. However, none of these models take the morphological parameters into factual consideration, while these parameters play an important role in the diagnostic phase.

In this retrospective and single-center study, we propose a novel morphological parameter, defined as M-ACT, as a new possible predictive marker of fibrotic evolution among Philadelphia-negative MPN. Furthermore, this new parameter seems to be useful to supplement WHO 2017 classification criteria in the differential diagnosis of the MPN subtype between ET and early/prefibrotic PMF.

In our study, carried out on a large cohort of MPN BM biopsies at diagnosis, extensive evidence support this statement. In fact, in univariate analysis M-ACT correlates with relevant MPN clinical and hematologic parameters (see Table 1 and 2), such as palpable splenomegaly, WBC or PLT count, and LDH levels, but also with molecular profiles defined by the JAK2 V617F allele burden and $C A L R$ mutations (especially the CALR type 1 mutation).

In PV patients the PFS was influenced at the multivariate analysis by the JAK2 V617F allele burden $>50 \%$, as already reported; ${ }^{26}$ in early/prefibrotic PMF patients' PFS was influenced by the presence of the CALR type 1 mutation, WBC count $>11 \times 10^{9} / \mathrm{L}$ and male sex, in agreement with previous reports. ${ }^{27}$

Moreover, patients with M-ACT had a significant correlation with a worse PFS and with an overt-myelofibrotic BM failure, in both PV and early/prefibrotic PMF $(P<0.0001)$. This last result is also confirmed at multivariate analysis. Interestingly, PV and early/prefibrotic PMF patients with this parameter showed a rapid clinical progression before the end of the 5-year follow-up, suggesting that M-ACT could be an early predictive marker capable of precociously identifying patients that need a closer follow-up.

Numerous scientific papers have highlighted that in the evolution towards myelofibrosis of MPN, a central role seems to be played by MK. Patients with MPN and fibrotic evolution showed a significantly increased number of MK with an abnormal nuclear/cytoplasmic ratio and a reduced polyploid state, often organized in clusters..$^{27,28}$ Experiments using in vitro cultures of $\mathrm{CD}_{3} 4^{+}$hematopoietic stem cells of patients with fibrotic MPN have shown that MK expand excessively, are immature and show delayed apoptosis owing to increased expression of the anti-apoptotic factor BCL-XL. ${ }^{29}$ Moreover, mice with a MK-specific deficiency of the transcription factor-encoding gene GATA1 show elevated numbers of immature MK in the BM and an increased and pathologic neutrophil emperipolesis that may represent one of the mechanisms leading to myelofibrosis by releasing fibrogenic $\mathrm{MK}$ cytokines and neutrophil proteases in the microenvironment of in vivo experiments. ${ }^{14,16}$ Finally, MK from individuals with PMF secrete increased levels of the fibrotic cytokines such as TGF- $\beta$, compared to MK from healthy individuals, and the extracellular matrix (ECM) microenvironment, especially the fibronectin component, is able to sustain progenitor cell proliferation and megakaryopoiesis in a TPO-independent manner. ${ }^{16,30,31}$ These pro-fibrotic cytokines would presumably act mainly in the microenvironment near to those MK clusters which are, in turn, their main producers. Furthermore, the criteria defining the megakaryocytic activation could represent the morphological counterpart of what is postulated by in vitro and in vivo studies regarding the role of $\mathrm{MK}$ in the $\mathrm{BM}$ fibrotic evolution of patients with MPN.

Recent evidence has suggested that treating patients with early-stage MF may lead to better outcomes with a less severe splenomegaly, a lower incidence of cytopenia, and less-severe BM fibrosis. However, the argument is debated, especially considerating the adverse events of the $J A K 2$ inhibitor treatment (ruxolitinib). M-ACT parameter, 
as an early predictive marker capable of precociously identifying patients with an overt-myelofibrotic BM failure, could also select those patients that would benefit from precocious treatment. ${ }^{32}$

Our analysis not only supports the role of the MK and their activation in the evolution of PV and early/prefibrotic PMF, but also seems to suggest that, for the treatment of this neoplasia, as well as the inhibition of specific mutations, which may partially alter the natural history of the disease, the blockage of the fibrotic evolution and therefore of its main key-player, the MK, should be a future therapeutic strategy to be investigated. ${ }^{30}$

$\mathrm{M}$-ACT is also a very useful morphological parameter in the diagnostic phase of MPN. In fact, none of the ET patients showed M-ACT, which when present, identifies only an early/prefibrotic PMF.

Interestingly the M-ACT showed a significant lower incidence in triple-negative patients in comparison to those with a driver gene mutation $(24.7 \%$ vs. $40.5 \%)$ reinforcing the idea of a more indolent disease for this subgroup while maintaining its predictive role for the fibrotic evolution also in triple-negative patients.

In addition, M-ACT parameter evaluation represents an easily executable analysis with a high agreement index between pathologists. Moreover, the search for $M$ ACT in BM biopsies in the diagnostic phase of MPN patients can be performed widely without the need for further analysis such as immunohistochemistry or molecular analysis.

The main limitation of our study is the retrospective design, hence the time estimate of progression to overt myelofibrosis and the estimate of PFS based on the patient chart review can lack of accuracy. Moreover, the team physician remained the same over years and the cri- teria for the diagnosing progression to overt myelofibrosis were aligned with those of the IWG-MRT consensus. $^{21}$

Although we analyzed a large cohort, the results of this single-center study need confirmation in other independent MPN patient cohorts, and M-ACT should be validated as a prognostic tool.

\section{Disclosures}

The authors have no conflict of interest to disclose. The study was carried out in accordance with the Declaration of Helsinki and the consent for retrospective analysis of all clinical data, according to the Ethical Committee of the Catholic University School of Medicine, was obtained by all the patients at the hospital admission. The report does not present identifying images or other personal or clinical details of participants that compromise anonymity.

\section{Contributions}

$L M L, M S, V F$ and $M M$ were the principal authors and the main contributors in writing the manuscript; $E R, S B, V R, M D C$ and $P C$ analyzed and interpreted the patient data; $M M, L M L$, $M S$ and VF performed the biopsies analysis; LML and VDS read and corrected the manuscript. All authors read and approved the final manuscript.

\section{Acknowledgments}

We thank Dr. Sara Capodimonti and Dr. Tonia Cenci for their technical support.

\section{Funding}

This study was supported by Università Cattolica del Sacro Cuore, Fondi d'Ateneo, Linea D1 (2018 and 2019; MM and $L M L)$.

\section{References}

1. Swerdlow SH, Campo E, Harris NL, Jaffe ES, Pileri SA, Stein H, Thiele J. WHO classification of tumours of haematopoietic and lymphoid tissues (Revised 4th edition). IARC: Lyon 2017.

2. Murphy S. Diagnostic criteria and prognosis in polycythemia vera and essential thrombocythemia. Semin Hematol. 1999;36(1 Suppl 2):S9-13.

3. Georgii A, Buesche G, Kreft A. The histopathology of chronic myeloproliferative diseases. Baillieres Clin Haematol. 1998;11(4):721-749.

4. Barbui T, Thiele J, Gisslinger $\mathrm{H}$, et al. The 2016 WHO classification and diagnostic criteria for myeloproliferative neoplasms: document summary and in-depth discussion. Blood Cancer J. 2018;8(2):15

5. Cervantes F, Dupriez B, Pereira A, et al. New prognostic scoring system for primary myelofibrosis based on a study of the International Working Group for Myelofibrosis Research and Treatment. Blood. 2009;113(13):2895-2901.

6. Passamonti F, Cervantes F, Vannucchi AM, et al. A dynamic prognostic model to predict survival in primary myelofibrosis: a study by the IWG-MRT (International Working Group for Myeloproliferative Neoplasms Research and Treatment). Blood. 2010;115(9):1703-1708.

7. Gangat N, Caramazza D, Vaidya R, et al. DIPSS plus: a refined Dynamic International Prognostic Scoring System for primary myelofibrosis that incorporates prognostic information from karyotype, platelet count, and transfusion status. J Clin Oncol. 2011:29(4):392-397.

8. Rumi E, Cazzola M. Diagnosis, risk stratification, and response evaluation in classical myeloproliferative neoplasms. Blood. 2017;129(6):680-692.

9. Grinfeld J, Nangalia J, Baxter EJ et al. Classification and personalized prognosis in myeloproliferative neoplasms. $N$ Engl J Med. 2018;379(15):1416-1430.

10. Guglielmelli P, Lasho TL, Rotunno G, et al. MIPSS70: mutation-enhanced international prognostic score system for transplantation-age patients with primary myelofibrosis. J Clin Oncol. 2018;36(4):310-318.

11. Tefferi A, Guglielmelli P, Lasho TL, et al. MIPSS70+ Version 2.0: mutation and karyotype-enhanced international prognostic scoring system for primary myelofibrosis. J Clin Oncol. 2018;36(17):1769-1770.

12. Tefferi A, Guglielmelli P, Nicolosi M, et al. GIPSS: genetically inspired prognostic scoring system for primary myelofibrosis. Leukemia. 2018;32(7):1631-1642.

13. Tefferi A, Guglielmelli P, Lasho TL, et al. Mutation-enhanced international prognostic systems for essential thrombocythaemia and polycythaemia vera. Br J Haematol. 2020;189(2):291-302.

14. Larocca LM, Heller PG, Podda G, et al. Megakaryocytic emperipolesis and platelet function abnormalities in five patients with gray platelet syndrome. Platelets. 2015;26 (8):751-757.
15. Schmitt A, Jouault H, Guichard J, Wendling F, Drouin A, Cramer EM. Pathologic interaction between megakaryocytes and polymorphonuclear leukocytes in myelofibrosis. Blood. 2000;96(4):1342-1347.

16. Centurione L, Di Baldassarre A, Zingariello $M$, et al. Increased and pathologic emperipolesis of neutrophils within megakaryocytes associated with marrow fibrosis in GATA-1(low) mice. Blood. 2004;104(12):3573-3580.

17. Yan XQ, Lacey D, Hill D, et al. A model of myelofibrosis and osteosclerosis in mice induced by overexpressing thrombopoietin (mpl ligand): reversal of disease by bone marrow transplantation. Blood. 1996;88(2): 402-409.

18. Gianelli U, Iurlo A, Vener C, et al. The significance of bone marrow biopsy and JAK2V617F mutation in the differential diagnosis between the "early" prepolycythemic phase of polycythemia vera and essential thrombocythemia. Am J Clin Pathol. 2008;130(3):336-342.

19. Ridgeway JA, Tinsley S, Kurtin SE. Practical guide to bone marrow sampling for suspected myelodysplastic syndromes. J Adv Pract Oncol. 2017;8(1):29-39.

20. De Stefano V, Ruggeri M, Cervantes F, et al High rate of recurrent venous thromboembolism in patients with myeloproliferative neoplasms and effect of prophylaxis with vitamin K antagonists. Leukemia. 2016; 30(10):2032-2038.

21. Barosi G, Mesa RA, Thiele J, et al. Proposed criteria for the diagnosis of post-poly- 
cythemia vera and post-essential thrombocythemia myelofibrosis: a consensus statement from the International Working Group for Myelofibrosis Research and Treatment. Leukemia. 2008;22(2):437-438.

22.Lee SH, Erber WN, Porwit A, Tomonaga M, Peterson LC. International council for standardization in hematology. ICSH guidelines for the standardization of bone marrow specimens and reports. Int $\mathrm{J} \mathrm{Lab}$ Hematol. 2008;30(5):349-364.

23. Vardiman JW, Thiele J, Arber DA, et al. The 2008 revision of the World Health Organization (WHO) classification of myeloid neoplasms and acute leukemia: rationale and important changes. Blood. 2009;114(5):937-951.

24.Afkhami M, Vergara-Lluri M, Brynes RK, Siddiqi IN. Peripheral blood smears, bone marrow aspiration, trephine and clot biopsies: methods and protocols. Methods Mol
Biol. 2014;1180:257-269.

25. Martini M, Cenci T, D'Alessandris GQ, et al. Epigenetic silencing of Id4 identifies a glioblastoma subgroup with a better prognosis as a consequence of an inhibition of angiogenesis. Cancer. 2013;119(5):10041012.

26.Passamonti F, Rumi E, Pietra D, et al. A prospective study of 338 patients with polycythemia vera: the impact of JAK2 (V617F) allele burden and leukocytosis on fibrotic or leukemic disease transformation and vascular complications. Leukemia. 2010;24(9):1574-1579.

27. Cerquozzi S, Tefferi A. Blast transformation and fibrotic progression in polycythemia vera and essential thrombocythemia: a literature review of incidence and risk factors. Blood Cancer J. 2015;5(11): e366.

28. Ciurea SO, Merchant D, Mahmud N, et al.
Pivotal contributions of megakaryocytes to the biology of idiopathic myelofibrosis. Blood. 2007;110(3):986-993.

29.Villeval JL, Cohen-Solal K, Tulliez M, et al High thrombopoietin production by hematopoietic cells induces a fatal myeloproliferative syndrome in mice. Blood. 1997;90(11):4369-4383.

30.Wen QJ, Yang Q, Goldenson B, et al. Targeting megakaryocytic-induced fibrosis in myeloproliferative neoplasms by AURKA inhibition. Nat Med. 2015;21(12):1473-1480.

31. Malara A, Gruppi C, Abbonante V, et al. EDA fibronectin-TLR4 axis sustains megakaryocyte expansion and inflammation in bone marrow fibrosis. J Exp Med. 2019;216(3):587-604.

32.Palandri F, Sabattini E, Maffioli M. Treating early-stage myelofibrosis. Ann Hematol. 2019;98(2):241-253. 